\title{
CAPACIDAdE DE CONTROLE DE SISTEMA DE CONTROLE DE ATITUDE DE VEÍCULOS LANÇADORES
}

\author{
DOMingos SÁlvio CARRIJO, WALDEMAR DE CASTRO LeITE FILHO. \\ Divisão de Sistemas Espaciais, Instituto de Aeronáutica e Espaço \\ Pça. Mal. Eduardo Gomes, 50, Vila das Acácias, 12.228-904, São José dos Campos - SP - Brasil \\ E-mails: domingosdscliae.cta.br, waldemarwclfliae.cta.br
}

\begin{abstract}
This paper describes the development of a method to quantify the control capability of the attitude control system of a launch vehicle under the action of external disturbances. The control capability is understood as the tracking capability of the commanded attitude, even in the presence of disturbances. Wind is considered the most significant external disturbance that acts on the launcher vehicle. Taking into account the aerodynamic and thrust conditions, the control forces and corresponding thrust deflection are computed, as required to counteract the action of wind gusts and wind shears. Thus, it is required that the control moment provided by thrust deflection can balance the maximum aerodynamic moment. Finally, data from a test case is presented, where a reference trajectory of a launch vehicle has been used.
\end{abstract}

Keywords: Dynamic Pressure, Static Margin, Aerodynamic Effectiveness, Control Capability, Movable Nozzle System.

\begin{abstract}
Resumo - Este artigo descreve o desenvolvimento de um método para quantificar a capacidade de controle de sistema de controle de atitude de veículos lançadores quando submetidos a perturbações externas. Entende-se por capacidade de controle a propriedade de rastreamento a uma atitude comandada e, alem disso, de manter controlado na presença de perturbações. O vento é considerado o distúrbio mais relevante que atua externamente no veículo. Com base nas características das cargas aerodinâmicas (pressão dinâmica e margem estática) e do empuxo, determinam-se as forças de controle geradas a partir da deflexão do jato principal de empuxo necessárias para equilibrar os efeitos das rajadas e o gradiente do vento. Espera-se que o momento de controle deve ser maior que o momento máximo de aerodinâmica. Os valores dos ângulos de deflexão apresentados foram calculados pelo método proposto, a partir de uma trajetória de referência de um veículo lançador exemplo.
\end{abstract}

Palavras-chave: Pressão Dinâmica, Margem Estática, Eficácia Aerodinâmica, Capacidade de Controle, Sistema Tubeira Móvel.

\section{Introdução}

A missão de veículos lançadores estabelece a definição de sua trajetória. Uma característica dinâmica relevante destes veículos é a de ter parâmetros variantes no tempo. Isto se deve ao fato de perder grande quantidade de massa, em função da queima do propelente ao longo do tempo, e dos parâmetros aerodinâmicos que variam com o número de Mach.

A partir da definição da trajetória, procura-se desenvolver algoritmos de controle de atitude eficientes, capazes de realizar a atitude programada e, além disso, de manter o controle quando sujeito a perturbações (vento, excentricidade do ponto de aplicação da força de empuxo e do centro de gravidade, assimetria e desalinhamento angular entre os valores de empuxos).

Os veículos lançadores geralmente têm suas forças de controle geradas a partir da deflexão do jato principal de empuxo através da utilização de dispositivos denominados de Sistema Tubeira Móvel - STM (Palmério, 2008).

O presente trabalho descreve o desenvolvimento de um método utilizado para quantificar o ângulo de deflexão do empuxo (definida como a capacidade de controle de sistema de controle de atitude) para controlar os efeitos dinâmicos devido ao vento (rajada e gradiente), considerado como o distúrbio mais relevante que atua externamente no veículo.

Para mostrar a aplicabilidade do método em questão, apresentam-se os valores dos ângulos de deflexão do empuxo (necessários para equilibrar os efeitos do vento), calculados a partir de uma trajetória de referência de um veículo lançador exemplo do Instituto de Aeronáutica e Espaço - IAE.

\section{Equação da Dinâmica Rotacional}

Este trabalho considera o veículo lançador em voo atmosférico mostrado na Figura 1 (Greensite, 1970; Palmerio, 2008, Kadam, 2009; Ramos, Leite Filho e Moreira, 2003), o qual é suposto completamente rígido.

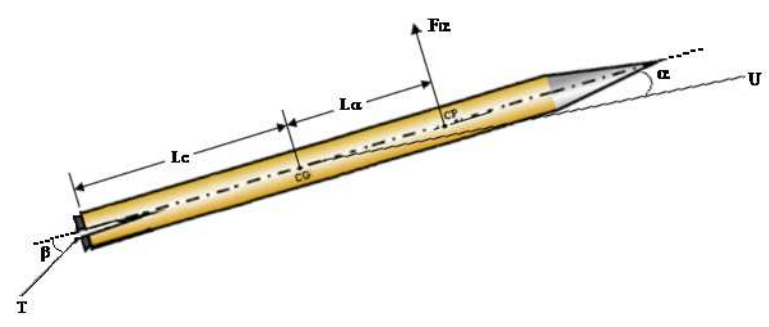

Figura 1: Veículo Lançador na Atmosfera

onde: 
$\mathrm{T}=$ força de empuxo;

$\mathrm{L}_{\mathrm{C}}=$ distancia do centro massa até ponto de aplicação do empuxo (braço de alavanca);

$\beta$ = ângulo de deflexão do empuxo;

$\mathrm{F}_{\alpha}=$ força aerodinâmica definida por

$$
\mathrm{F}_{\alpha}=\mathrm{C}_{\mathrm{N} \alpha} \cdot \operatorname{Pdin} \mathrm{Ar} \cdot \alpha
$$

$\mathrm{C}_{\mathrm{N \alpha}}=$ coeficiente aerodinâmico;

Pdin = pressão dinâmica;

$\mathrm{Ar}=$ área de referência;

$\mathrm{L}_{\alpha}=$ distancia do centro massa até centro de pressão (margem estática); $\mathrm{e}$

$\alpha=$ ângulo de ataque.

Com base na equação de momento (Greensite, 1970), tem-se

$$
\mathrm{I}_{\mathrm{yy}} \cdot \ddot{\theta}=\sum \mathrm{M}_{\mathrm{y}}
$$

ou seja,

$$
\mathrm{I}_{\mathrm{yy}} \ddot{\theta}=\mathrm{T} \cdot \mathrm{L}_{\mathrm{C}} \cdot \beta+\mathrm{F}_{\alpha} \cdot \mathrm{L}_{\alpha}
$$

com $\ddot{\theta}=$ aceleração angular.

Substituindo-se a definição de $F_{\alpha}$ (1) em (3), obtém-se

$$
\ddot{\theta}=\frac{T \cdot L_{C}}{I_{y y}} \cdot \beta+\frac{C_{\mathrm{N} \alpha} \cdot P d i n \cdot A r \cdot L_{\alpha}}{I_{y y}} \cdot \alpha
$$

Na sequencia, define-se a eficácia de controle da força de empuxo $\mu_{\beta}$ (Giannini e Cruciani, 2013) dado por

$$
\mu_{\beta}=\frac{T \cdot L_{C}}{I_{y y}}
$$

e a eficácia aerodinâmica $\mu_{\alpha}$ (Palmerio, 2008; Giannini e Cruciani, 2013) como se segue

$$
\mu_{\alpha}=\frac{\mathrm{C}_{\mathrm{N \alpha}} \cdot \operatorname{Pdin} \cdot \operatorname{Ar} \cdot \mathrm{L}_{\alpha}}{\mathrm{I}_{\mathrm{yy}}}
$$

Assim sendo, substituindo-se (5)-(6) em (4) obtém-se a equação da dinâmica rotacional simplificada (Ramos, Leite Filho e Moreira, 2003) na forma

$$
\ddot{\theta}=\mu_{\beta} \cdot \beta+\mu_{\alpha} \cdot \alpha
$$

\section{Trajetória de Referência}

Para mostrar a aplicabilidade do método a ser desenvolvido a fim de quantificar a capacidade de controle devido os efeitos dinâmicos do vento, apresentam-se os seguintes dados temporais da trajetória referência de um veículo exemplo

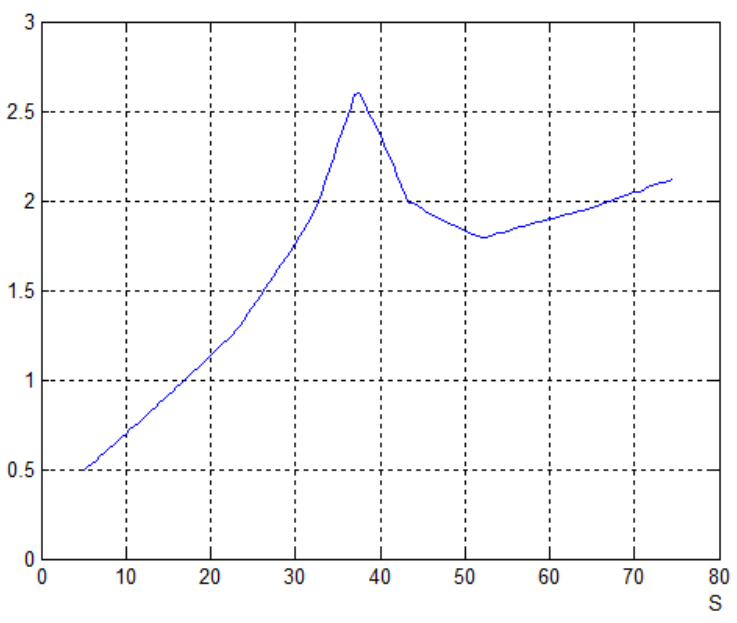

Figura 2: Margem Estática (m)

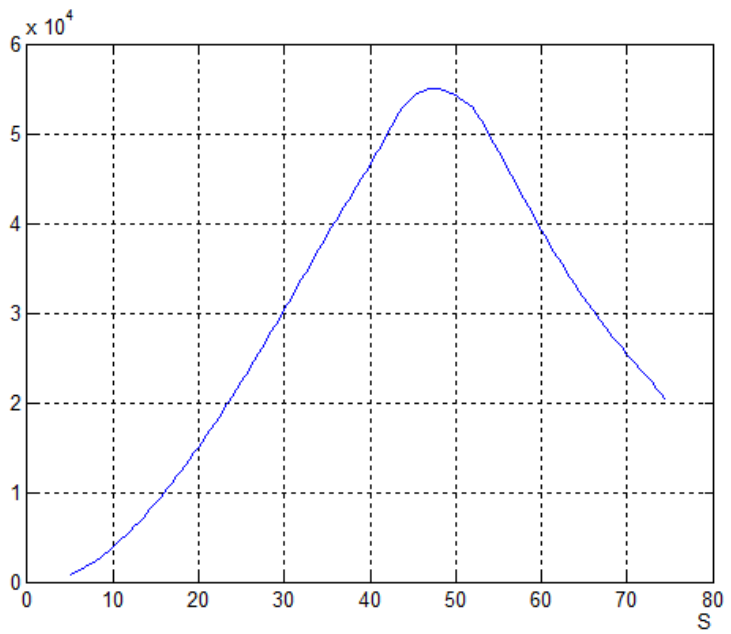

Figura 3: Pressão Dinâmica (Pa)

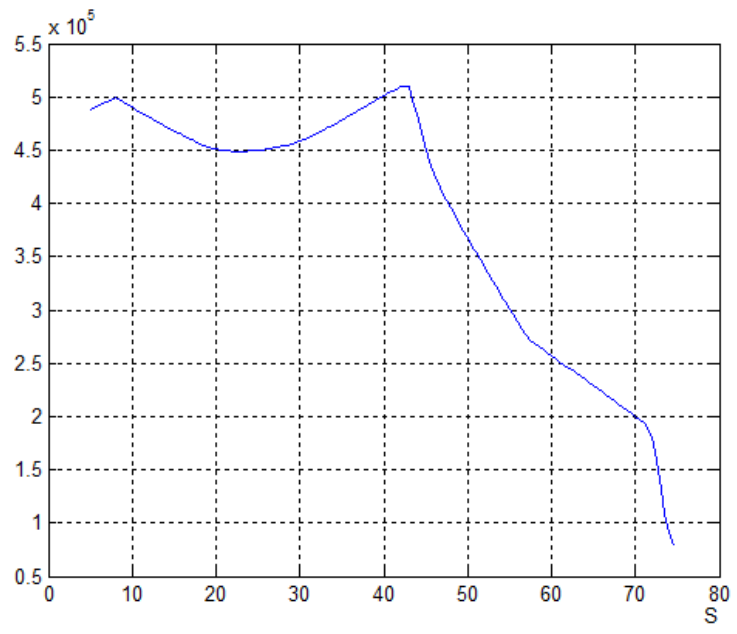

Figura 4: Força de Empuxo (N) 


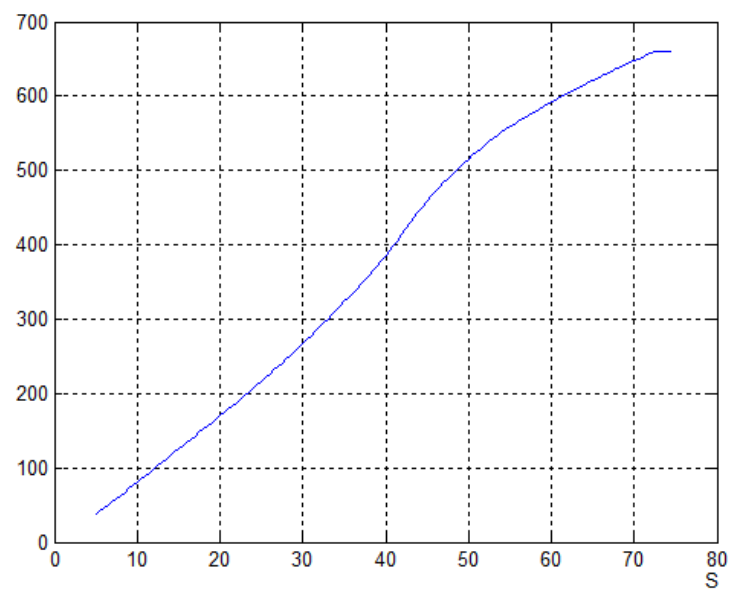

Figura 5: Velocidade Longitudinal $(\mathrm{m} / \mathrm{s})$

Com os dados da trajetória de referência e (5) obtém-se

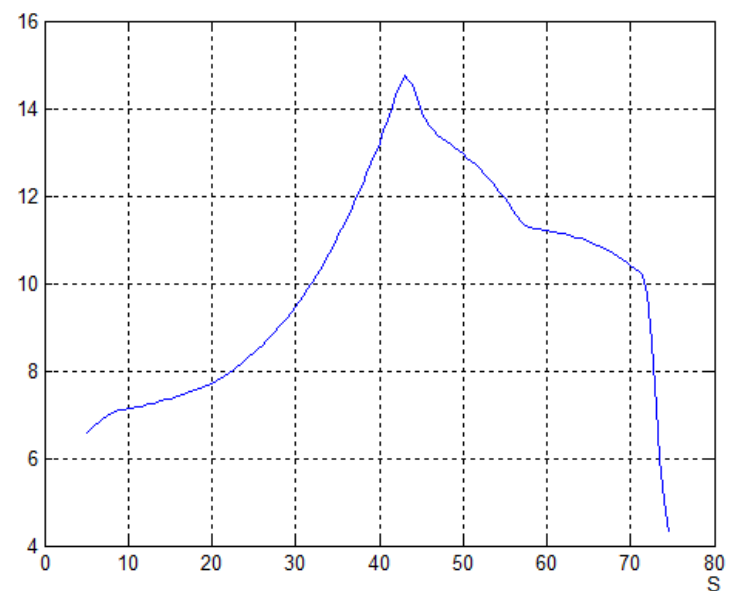

Figura 6: Eficácia de Controle da Força de Empuxo $\mu_{\beta}$

e, ainda, a partir de (6), resulta

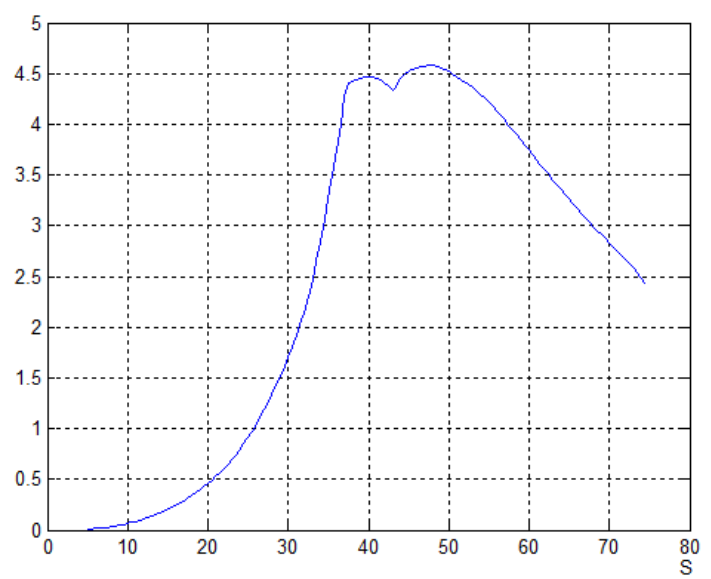

Figura 7: Eficácia Aerodinâmica $\mu_{\alpha}$

\section{4 Ângulo de Deflexão do Empuxo}

Como a capacidade de controle de um veículo lançador é definida pela propriedade de rastreamento a uma atitude comandada e de se manter controlado quando sujeito a conjunto de perturbações (vento, excentricidade do ponto de aplicação da força de empuxo e do centro de gravidade, assimetria e desalinhamento angular entre os valores de empuxos). Neste trabalho, considera-se o vento como o distúrbio mais relevante que atua no veículo.

Então, a capacidade de controle é quantizada pelo ângulo de deflexão do empuxo necessário para controlar os efeitos devido as rajadas (Wind Gust) e o gradiente de vento (Wind Shear), considerados como fenômenos aleatórios.

O ângulo de deflexão para controlar o efeito da rajada de vento é encontrado a partir de (7) estaticamente, ou seja, considerando que o veiculo controlado deve ser capaz de atingir $\ddot{\theta}=0$ o que implica em $\dot{\theta}=\dot{\theta}_{\mathrm{R}}$ e $\theta=\theta_{\mathrm{R}}$ (valores da trajetória de referência). Desta forma, estabelecendo-se um limite superior para o ângulo de ataque $\alpha$ (ou seja, $\alpha<\alpha_{\text {MAX }}$ ) obtém-se

$$
\beta>\frac{\left|\mu_{\alpha}\right|}{\mu_{\beta}} \cdot \alpha_{\mathrm{MAX}}
$$

Com $\alpha_{\mathrm{MAX}} \approx$ Vraj/U (Vraj é o vento de rajada e U a velocidade longitudinal), então de (8) pode-se afirmar que o ângulo de deflexão necessário para controlar o vento de rajada é dado por

$$
\beta r a j>\frac{\left|\mu_{\alpha}\right|}{\mu_{\beta}} \cdot \frac{\text { Vraj }}{U}
$$

Na seqüência, o ângulo de deflexão para controlar o efeito do gradiente é obtido de forma dinâmica ao considerar $\ddot{\alpha} \cong \ddot{\theta}$ em (7), resultando-se

$$
\ddot{\alpha} \cong \mu_{\beta} \cdot \beta+\mu_{\alpha} \cdot \alpha
$$

Aplicando-se a Transformada de Laplace em (10), pode-se expressar o que se segue

$$
\dot{\alpha}(s)=\frac{\mu_{\beta} \cdot s}{s^{2}-\mu_{\alpha}} \beta(s)
$$

Com base no Teorema de Parseval (Cook, 1994), permite fazer o seguinte desenvolvimento

$$
\begin{aligned}
\sup _{\beta} \frac{\|\dot{\alpha}\|}{\|\beta\|} & ={ }_{\omega}^{\sup } \mid G(j \omega \mid \\
& =\sup _{\omega}\left|\frac{j \omega \mu_{\beta}}{-\omega^{2}-\omega_{\alpha}}\right| \\
& =\frac{\mu_{\beta}}{2 \cdot \sqrt{\mu_{\alpha}}}
\end{aligned}
$$

De (11)-(12), resulta que 


$$
\beta \geq \dot{\alpha}_{\mathrm{MAX}} \cdot 2 \cdot \frac{\sqrt{\mu_{\alpha}}}{\mu_{\beta}}
$$

Agora, supondo um valor máximo para o gradiente de vento (ou seja, Vgrad $\leq \dot{\alpha}_{\text {MAX }}$ ), resulta que o ângulo de deflexão necessário para controlar seu efeito pode ser descrito na forma

$$
\beta \operatorname{grad} \geq \frac{2 . \operatorname{Vgrad} \cdot \sqrt{\left|\mu_{\alpha}\right|}}{\mu_{\beta}}
$$

\section{Resultados Obtidos}

De (5)-(6) e (9) e adotando-se o valor de vento de rajada Vraj $=27,8558 \mathrm{~m} / \mathrm{s}$, obtém-se

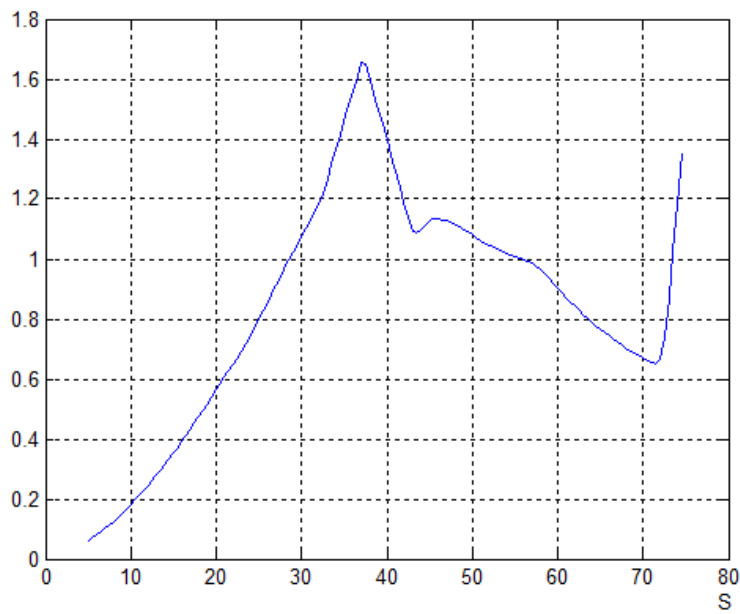

Figura 8: Angulo de Deflexão Devido Vento de Rajada (graus)

Usando (5)-(6) e (14) com o valor do gradiente de vento $\operatorname{Vgrad}=0,05793 / \mathrm{s}$, tem-se

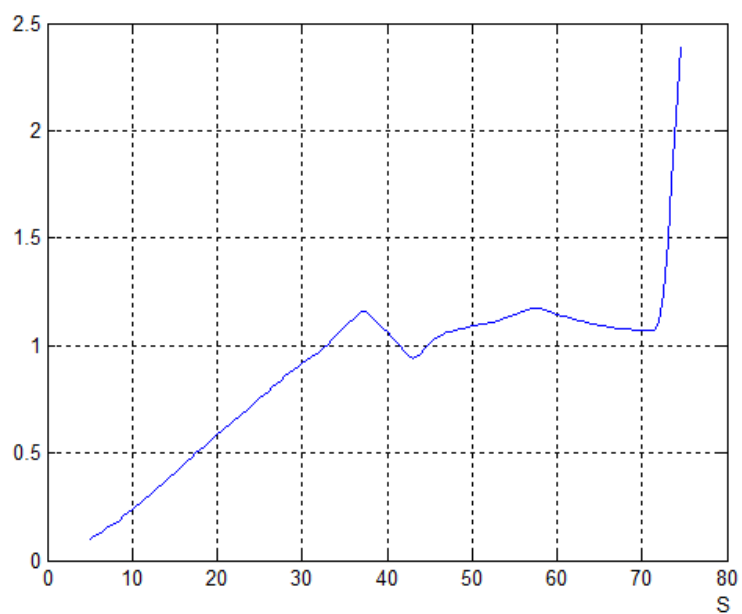

Figura 9: Angulo de Deflexão Devido Gradiente de Vento (graus)

Uma vez que, as rajadas e o gradiente de vento são considerados fenômenos aleatórios (valores máximos obtidos em 3 vezes o desvio padrão) e como os valores de deflexão do empuxo $\beta$ raj (9) e $\beta$ grad (14) são calculados separadamente, então sua soma deve ser feita como variância (soma dos quadrados) na forma de

$$
\beta \text { vento }=\sqrt{\beta \mathrm{raj}^{2}+\beta \operatorname{grad}^{2}}
$$

obtendo-se o que se segue

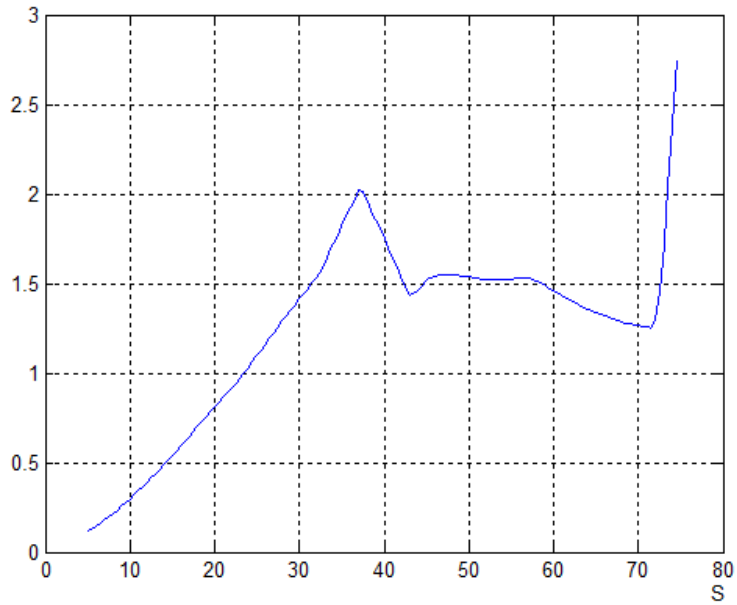

Figura 10: Angulo de Deflexão Devido ao Vento (graus)

\section{Comentários e Conclusões}

Observa-se que o ângulo de deflexão do empuxo necessário para compensar as perturbações do vento considerados neste trabalho esta em torno de 2,1 graus (ver Figura 10) obtidos para região de pressão dinâmica máxima (ver Figura 3), portanto, menor do que os valores máximos adotados pelos fabricantes de STM que é de 4,0 graus. É importante ressaltar que os valores considerados para o vento sugerem o pior caso, pouco provável que ocorra na realidade.

Outro fato importante que merece comentário é em relação ao polo de (11) dado por $\sqrt{\mu_{\alpha}}$. Nota-se que este estabelece um limite inferior para o valor da banda passante do STM a ser usado. Na prática, deve ser adotado um valor maior que $6 \cdot \sqrt{\mu_{\alpha}}$.

\section{Referências Bibliográficas}

Cook, P. A. (1994). Nonlinear Dynamical Systems. Englewood Cliffs, Prentice-Hall, New Jersey.

Giannini, M. e Cruciani, I. (2013). VEGA LV Qualification Process: GNC aspects on HWIL Testing and Analysis. EUCASS 2013, $5^{\text {th }}$ European Conference for Aeronautics and Space Sciences, Munique.

Greensite, A. L. (1970). Analysis and Design of Space Vehicle Flight Control System. Spartan Books, Washington, New York.

Kadam, N. V. (2009). Practical Design of Flight Control System for Launch Vehicles and Missiles. Allied Publishers Private Limited, New Dellhi.

Palmerio, A. F. (2008). Introdução à Tecnologia de Foguetes. MPEA, ITA, São José dos Campos.

Ramos, F. O.; Leite Filho, W. C. e Moreira, F. J. O. (2003). Gain Computational Strategy for na Attitude Control System. COBEM 2003, $17^{\text {th }}$ International Congress of Mechanical Engineering, São Paulo. 